\title{
Life-Saving Combined Heart-Kidney Transplantation in a Previous Sequential Heart and Kidney Transplant Recipient
}

\author{
Meenal Sharma ${ }^{1}$, Chris Anthony ${ }^{1}$, Christopher Hayward ${ }^{1}$, Andrew Jabbour ${ }^{1}$, Anne M Keogh ${ }^{1}$, Peter Macdonald ${ }^{1}$, Jacob Sevastos ${ }^{2}$ \\ ${ }^{1}$ Heart \& Lung Transplant Unit, St Vincent's Hospital Sydney, New South Wales, Australia \\ ${ }^{2}$ Department of Nephrology, St Vincent's Hospital Sydney, New South Wales, Australia
}

Doi: 10.12890/2018_000924- European Journal of Case Reports in Internal Medicine - ๔ EFIM 2018

Received: 03/06/2018

Accepted: 09/06/2018

Published: $19 / 06 / 2018$

How to cite this article: Sharma M, Anthony C, Hayward C, Jabbour A, Keogh AM, Macdonald P, Sevastos J. Life saving combined heart-kidney transplantation in a previous sequential heart and kidney transplant recipient. EJCRIM 2018;5: doi:10.12890/2018_000924.

Conflicts of Interests: The Authors declare that there are no competing interests.

Acknowledgements: The Authors thank Khumud Dhital, Emily Granger, Paul Jansz and Eugene Kotlyar for their contribution to the paper.

This article is licensed under a Commons Attribution Non-Commercial 4.0 License

\section{ABSTRACT}

Purpose: Solid organ re-transplantation in the context of allograft failure is a challenging clinical and ethical problem. Ideally, solid organ retransplantation after initial allograft failure should be performed in all recipients, but this is often not clinically or logistically feasible.

Methods: This report details what we believe is the first combined heart-kidney transplant in a recipient of a previous sequential heart and kidney transplant.

Results: Eight years after a combined heart and kidney transplant after initially receiving a sequential heart and kidney transplant, a 31-yearold man is doing extremely well, with no rejection episodes or significant complications after transplantation.

Summary: This case confirms that combined heart and kidney transplantation is a viable option for tackling the complex issue of graft failure in recipients of previous cardiac and renal grafts.

\section{LEARNING POINTS}

- Good short- and long-term outcomes following combined heart-kidney transplantation can be achieved in patients with multi-system end-organ dysfunction.

- Advances in immunosuppressant therapy have enabled multiple transplantation procedures from different donors in a single recipient.

- We describe the first recipient in the world of combined heart-kidney transplantation on a background of previous sequential heartkidney transplantation.

\section{KEYWORDS}

Heart kidney transplantation; combined heart kidney transplantation; recipient of multiple transplanted organs

\section{INTRODUCTION}

End-stage cardiac and renal dysfunction often co-exist given the direct correlation and interaction between these organs. The poor outcomes of cardiac transplant recipients who require dialysis are well described ${ }^{[1]}$ and multiorgan dysfunction in itself is a contraindication for transplantation given the high mortality rates in this cohort of patients ${ }^{[2]}$. Combined heart-kidney transplantation is a viable solution for patients with end-stage cardiac and renal dysfunction who otherwise would not be able to receive single organ transplants due to multiorgan dysfunction ${ }^{[3,4]}$. We describe what we believe is the first case in the world of combined heart-kidney transplantation in a recipient of a previous sequential heart and kidney transplant. 


\section{CLINICAL CASE}

A 31-year-old man underwent successful orthotopic heart transplantation in 1990 at the age of 7 for end-stage heart failure due to familial dilated cardiomyopathy. He subsequently developed chronic kidney disease attributed to calcineurin inhibitor nephrotoxicity which progressed to end-stage renal impairment necessitating renal replacement therapy in 2003. Due to his quick progression from peritoneal dialysis to haemodialysis, the decision was made that he should undergo cadaveric renal transplantation in 2004. He was HLA typed and found to be HLA A2, A24, B39, B60, DR4, DR13, while his renal donor was HLA A2, homogenous B60, B62, DR4, DR13. Incidentally, his initial donor heart was HLA A2, A24, B39, B60, DR4, DR13. His blood group was AB+.

He underwent successful cadaveric renal transplantation but 4 years later developed severe cardiac allograft vasculopathy with two ST-elevation myocardial infarcts necessitating percutaneous coronary intervention for a $70 \%$ left main lesion and an $80 \%$ right coronary artery lesion. His now ischaemic allograft cardiomyopathy left him with poor cardiac graft function with an ejection fraction of $25 \%$. The combination of acute ischaemic insult after infarct, ischaemic cardiomyopathy and ongoing calcineurin inhibitor use resulted in cardiorenal syndrome and allograft vasculopathy, leading to worsening of his renal function. He subsequently required renal replacement therapy again and was on peritoneal dialysis in 2009.

Due to his worsening cardiac and renal graft function, he was placed on the transplant list for a combined heart and kidney transplant at the time of his renal replacement initiation, and received a non-HLA matched combined heart and kidney transplant in 2010. Eight years after receiving the combined heart and kidney transplant, he is doing extremely well, with no rejection episodes or significant complications, and leads an active and normal life.

\section{DISCUSSION}

This case demonstrates the utility of combined heart-kidney transplantation as a viable option for patients with dual cardiac and renal endorgan dysfunction, and in this unique circumstance, combined heart-kidney transplantation in a previous recipient of sequential heart and kidney transplantation.

Irrespective of potential sensitisation to multiple previous donor antibodies, it is a testament to the viability of multiple transplantation procedures in a single recipient due to the established effectiveness of immunosuppressant therapy and donor antibody matching in the modern era ${ }^{[5]}$.

\section{REFERENCES}

1. Alam A, Badovinac K, Ivis F, Trpeski L, Cantarovich M. The outcome of heart transplant recipients following the development of end-stage renal disease: analysis of the Canadian Organ Replacement Register (CORR). Am J Transplant 2007;7:461-465.

2. Cassuto JR, Reese PP, Sonnad S, et al. Wait list death and survival benefit of kidney transplantation among nonrenal transplant recipients. Am J Transplant 2010;10:2502-2511.

3. Gill J, Shah T, Hristea I, et al. Outcomes of simultaneous heart-kidney transplant in the US: a retrospective analysis using OPTN/UNOS data. Am J Transplant 2009;9:844-852.

4. Russo MJ, Rana A, Chen JM, et al. Pretransplantation patient characteristics and survival following combined heart and kidney transplantation: an analysis of the United Network for Organ Sharing Database. Arch Surg 2009;144:241-246.

5. Lindenfield, J, Miller GG, Shakar SF, Zolty R, et al. Drug therapy in the heart transplant recipient part 1: cardiac rejection and immunosuppressive drugs. Circulation 2004;110:3734-3740. 